\title{
28 Research Soure \\ Oropharyngeal dysphagia and quality of life in elderly patients after stroke.
}

\section{Leticia Sampaio de Oliveira}

Bauru School of Dentistry, University of São Paulo

Jonan Emi Valencia Cardenas ( $\boldsymbol{\sigma}$ jvalencia@usp.br)

University of Sao Paulo https://orcid.org/0000-0002-2115-9980

Claudia Tiemi Mituuti

Federal University Santa Catarina

\section{Raquel Rodrigues Rosa}

Bauru School of Dentistry, University of São Paulo

\section{Eduardo Carvalho de Andrade}

Universidade de Sao Paulo Faculdade de Odontologia de Bauru

Giédre Berretin-Felix

Bauru School of Dentistry, University of São Paulo

\section{Research article}

Keywords: dysphagia, elderly, quality of life, stroke

Posted Date: November 8th, 2019

DOI: https://doi.org/10.21203/rs.2.17064/v1

License: (c) (i) This work is licensed under a Creative Commons Attribution 4.0 International License.

Read Full License 


\section{Abstract}

Objective To verify the association between oropharyngeal dysphagia and quality of life in elderly individuals, at the late stage of stroke.

Methods Retrospective cross-sectional study, whose results were obtained by analysis of the databank of a project that analyzed 30 elderly individuals affected by stroke in the late stage. All were submitted to clinical and instrumental evaluation (fiberoptic endoscopic) of swallowing, testing foods of solid, liquid and pudding textures. The degree of dysphagia was classified according to data from clinical examination and images of videoendoscopy examinations using the DOSS scale (Dysphagia Outcome and Severity Scale). The quality of life related to swallowing was analyzed by the SWAL-QOL protocol (Quality of Life in Swallowing Disorders), previously applied to the elderly individuals. Data were submitted to descriptive statistical an analysis and the Spearman correlation test.

Results The clinical evaluation evidenced that most individuals presented mild oropharyngeal dysphagia, while the DOSS scale demonstrated swallowing with functional limitations. Mild impact or lack of impact was observed on the quality of life related to swallowing. There was positive correlation between burden, wish to feed, duration of feeding and mental state with the severity of oropharyngeal dysphagia, both by clinical and instrumental evaluations; and between the frequency of symptoms and severity of dysphagia by clinical evaluation $(p<0.01)$.

Conclusion There was association between the severity of oropharyngeal dysphagia and quality of life of elderly individuals in the late stage of stroke, demonstrating the importance of longitudinal follow-up of these individuals concerning the feeding aspects.

\section{Introduction}

Dysphagia is characterized by the difficulty to form and transport the food bolus from the mouth to the stomach in a safe and efficient manner. Besides the impact on the quality of life, it is associated with respiratory complications and affects the nutritional status, being that most patients are not diagnosed and treated properly ${ }^{(1)}$.

In the adult and elderly population, dysphagia is more commonly associated with stroke. More than half of patients who suffered stroke have six to ten types of disability, including neurological dysphagia ${ }^{(2)}$.

By clinical evaluation, authors state that adult and elderly patients affected by both ischemic and hemorrhagic stroke present high rate of oropharyngeal dysphagia, characterized by laryngeal penetration $(3-4-5-6-7)$. However, most patients have little or no knowledge about their swallowing dysfunction ${ }^{(3)}$.

Based on validated protocols, it was observed that half of patients after the acute stage of stroke present at least one moderate neurological deficit ${ }^{(5)}$, with higher prevalence of moderate dysphagia, with signs of laryngeal penetration and presence of cough ${ }^{(8)}$. 
In the presence of late stroke, i.e. the non-acute stage, clinical evaluation revealed that nearly half of patients present some degree of dysphagia, and there may be significant relationship between the severity of stroke and the presence of dysphagia, and these patients also present high mortality rate due to aspiration pneumonia ${ }^{(7)}$.

The incidence of dysphagia increases in studies that include instrumental evaluation ${ }^{(9)}$. This technique is more appropriate to determine the risk of aspiration in acute ${ }^{(10)}$ and late stroke ${ }^{(11)}$. The greater sensitivity of instrumental examination allows to detect both mild forms of dysphagia and alterations in the pharyngeal phase, which are hardly observed by clinical evaluation ${ }^{(9)}$.

The quality of life is also an important factor to be investigated in patients after stroke with swallowing dysfunction, since it may aid the speech-language pathologist intervention and provide the patient with better swallowing function, greater wellbeing during feeding and benefits in other aspects of life.

Several studies in the literature employed the SWAL-QOL protocol (Quality of Life in Swallowing Disorders - SWAL-QOL) ${ }^{(12)}$, a specific questionnaire that addresses the quality of life related to swallowing to determine the impact of swallowing dysfunction on the quality of life of individuals with different conditions such as laryngectomized ${ }^{(13)}$, with Parkinson disease ${ }^{(14)}$, patients with amyotrophic lateral sclerosis $^{(15)}$ and after stroke ${ }^{(16-17)}$. Most studies have included adult and elderly individuals after the acute stage of stroke, and no studies were found in the literature investigating the correlation between the degree of dysphagia and quality of life using the SWAL-QOL protocol. Thus, this study aimed to verify the association between the degree of oropharyngeal dysphagia and the quality of life of elderly individuals after the late stage of stroke.

It is believed that investigation of the quality of life may aid to understand and observe the signs and symptoms of oropharyngeal dysphagia in each individual and also provide subsides to select the most adequate treatment to enhance the swallowing and consequently the quality of life of each individual.

\section{Methods}

This retrospective cross-sectional study was conducted by analysis of a databank of a Speech-Language Pathology Clinic, and was initiated after approval by the Institutional Review Board, protocol n. 553.710 and CAEE n. 16365913.0000.5417.

The study collected information from 30 elderly individuals affected by stroke with age between 61 and 90 years (mean=74.4 and standard deviation=9.6 years), being 17 males and 13 females who met the following inclusion criteria: medical diagnosis of stroke with time ranging from 6 to 108 months, respecting the maximum time of spontaneous 
recovery ${ }^{(18)}$; under regular clinical neurological follow-up; informing stable general health condition for accomplishment of the proposed examinations ${ }^{(18)}$ and presenting minimum score of 18/19 points on application of the Mini Mental State Examination (19-20). The study excluded individuals with history of other associated neurological or oncological diseases.

For evaluation of swallowing, three standardized food textures were used and offered in the following order:

- Pudding $(10 \mathrm{ml}): 30 \mathrm{ml}$ of filtered water added to $2 \mathrm{~g}$ of powdered diet grape juice (Clight) thickened with one spoon of spontaneous thickener (NUTILIS, Support), prepared with a disposable spoon with 10-ml capacity;

- Solid: one 1-cm thick slice of bread roll with approximately 4-cm diameter. It should be mentioned that this bread was necessarily baked on the same day and period of evaluation and was always purchased from the same bakery;

- Liquid (10 ml): filtered water collected with a 10-ml syringe and offered in a plastic cup (with $50 \mathrm{ml}$ capacity).

The individuals were asked to place the liquid and pudding contents in the mouth and swallow voluntarily. Concerning the solid food offered, the individual was asked to place the bread in the mouth and voluntarily chew, organize and swallow the food bolus. The clinical evaluation was performed by a speech-language pathologist with training in the field of oropharyngeal dysphagia. The degree of dysphagia was scored considering the results of this evaluation and pulse oximetry - oxygen saturation (SpO2) to confirm the signs of aspiration. Thus, the individuals were divided into three levels according to the degree of swallowing involvement, as presented in Chart 1 below ${ }^{(21)}$. 


\begin{tabular}{|l|l|l|}
\hline \multicolumn{2}{|c|}{ Classification } & \multicolumn{1}{c|}{ Observed aspects } \\
\hline 1 & Mild & $\begin{array}{l}\text { When the control and transportation of the food bolus is delayed and slow, without signs of laryngeal } \\
\text { penetration on cervical auscultation. There is alteration of the lip sphincter, tongue incoordination, delay to } \\
\text { trigger the swallowings reflex, absence of cough, without marked reduction of laryngeal elevation, without } \\
\text { alteration of vocal performance after swallowings and normal cervical auscultation. }\end{array}$ \\
\hline 2 & Moderate & $\begin{array}{l}\text { When the control and transportation of the food bolus is delayed and slow, with signs of laryngeal penetration } \\
\text { and risk of aspiration. There is alteration of the lip sphincter, tongue incoordination, delay or absence of } \\
\text { swallowing reflex, absence of cough, presence of cough before, during or after swallowing, reduction in } \\
\text { laryngeal elevation, alteration in vocal performance after swallowing and altered cervical auscultation. }\end{array}$ \\
\hline 3 & Severe & $\begin{array}{l}\text { When there are signs of aspiration confirmed by oximetry and absence or failure in complete swallowing of the } \\
\text { food bolus. There is delay or absence of swallowing reflex, reduction in laryngeal elevation, absence of cough, } \\
\text { presence of cough during or after swallowing, alteration of vocal performance after swallowing, evident } \\
\text { respiratory alteration, altered cervical auscultation and incomplete swallowing. }\end{array}$ \\
\hline
\end{tabular}

Chart 1: Classification of the degree of oropharyngeal swallowing dysfunction based on clinical evaluation of swallowing.

The instrumental evaluation (fiberoptic endoscopic evaluation of swallowing) was performed by an otolaryngologist and conducted by a speech-language pathologist. During examination, care was taken to avoid interference from the examiner. However, at completion of the swallowing process performed by the individuals for each texture, guidance or suggestions of postural maneuvers were provided, essentially, for patients presenting food stasis or airway permeation. The maneuvers suggested during examination included: head folding or chin downwards, swallowing with effort and multiple swallowing.

To analyze the examinations, three professionals with at least one year of experience in the field of oropharyngeal dysphagia were invited to be judges (all signed an invitation letter for participation in the study) and scored the degree of dysphagia according to the Dysphagia Outcome and Severity Scale - DOSS ${ }^{(22)}$, which scores the degree of dysphagia into levels ranging from 7 (normal in all situations) to 1 (severe dysphagia: nothing by oral feeding: unable of safe oral feeding). The agreement between examiners was analyzed by the kappa test and, according to its interpretation, it reached $100 \%$ between examiners 1 and 2 ( $\mathrm{k}=1.00$; excellent), 86.7\% between examiners 2 and 3 and between examiners 1 and $3(\mathrm{k}=0.71$; substantial). The mode or consensus between examiners were considered as final result. 
Concerning the quality of life, information contained in the SWAL-QOL protocol (Quality of Life in Swallowing Disorders - SWAL-QOL) ${ }^{(12-13-14-23)}$ were analyzed.

The results were analyzed by descriptive statistics and the Spearman correlation test to verify the relationship between the classification of oropharyngeal dysphagia and scoring in the quality of life protocol related to swallowing. All tests considered a significance level of $0.05 \%$.

\section{Results}

Clinical evaluation of swallowing revealed that most individuals presented mild oropharyngeal dysphagia (Graphic 1), and instrumental evaluation (fiberoptic endoscopic evaluation of swallowing) evidenced that most individuals scored grade 6 on the DOSS scale, i.e. with independent functional limitations/modifications (Graphic 1).

Based on data collected from the SWAL-QOL quality of life protocol (Table 1), it was observed that, in all domains, most patients presented maximum score, close or equal to 100 points, while the minimum score was close to 0 point for few individuals and few domains. The same was observed on analysis of the minimum and maximum global scores, in which the maximum was close to 100 points and the minimum was close to half of the maximum score.

Analysis of the association between the degree of dysphagia on the clinical and instrumental evaluation and the quality of life in each domain of the protocol is presented in Table 2. For clinical evaluation, there was moderate negative correlation between the degree of dysphagia and the domains frequency of symptoms (liquid texture), wish to feed, frequency of symptoms and global score (pudding texture) and burden, duration of feeding, frequency of symptoms and mental state (solid texture), demonstrating that the worse the degree of dysphagia, the greater the impact on the quality of life of these individuals. Concerning the correlation between the degree of dysphagia based on the instrumental evaluation and quality of life, there was statistically significant moderate positive correlation between the results of DOSS and the domains: burden, wish to feed, frequency of symptoms and mental state of the protocol, besides correlation with the global score, demonstrating that the worse the swallowing dysfunction in these cases, the greater was the impact on the quality of life related to swallowing.

Clinical evaluation of swallowing revealed that most individuals presented mild oropharyngeal dysphagia (Graphic 1), and instrumental evaluation (fiberoptic endoscopic evaluation of swallowing) evidenced that most individuals scored grade 6 on the DOSS scale, i.e. with independent functional limitations/modifications (Graphic 1).

Based on data collected from the SWAL-QOL quality of life protocol (Table 1), it was observed that, in all domains, most patients presented maximum score, close or equal to 100 points, while the minimum 
score was close to 0 point for few individuals and few domains. The same was observed on analysis of the minimum and maximum global scores, in which the maximum was close to 100 points and the minimum was close to half of the maximum score.

Analysis of the association between the degree of dysphagia on the clinical and instrumental evaluation and the quality of life in each domain of the protocol is presented in Table 2. For clinical evaluation, there was moderate negative correlation between the degree of dysphagia and the domains frequency of symptoms (liquid texture), wish to feed, frequency of symptoms and global score (pudding texture) and burden, duration of feeding, frequency of symptoms and mental state (solid texture), demonstrating that the worse the degree of dysphagia, the greater the impact on the quality of life of these individuals. Concerning the correlation between the degree of dysphagia based on the instrumental evaluation and quality of life, there was statistically significant moderate positive correlation between the results of DOSS and the domains: burden, wish to feed, frequency of symptoms and mental state of the protocol, besides correlation with the global score, demonstrating that the worse the swallowing dysfunction in these cases, the greater was the impact on the quality of life related to swallowing.

\section{Discussion}

This study investigated the association between the severity of oropharyngeal dysphagia in elderly individuals in the late stage of stroke and their quality of life.

The results demonstrated that most individuals, in the clinical evaluation, were classified as presenting mild oropharyngeal dysphagia for all food textures analyzed. Studies analyzing the incidence of dysphagia in elderly individuals in both acute ${ }^{(3,4)}$ and late ${ }^{(7)}$ stages of stroke observed that more than $50 \%$ of this population presents oropharyngeal dysphagia, which may range from mild, usually in the late stage, moderate or severe in the acute stage, according to the clinical evaluation of swallowing. It should be considered that, in the late stage of stroke, there may be a reduction in feeding difficulties, since these individuals find different manners to adapt their feeding, yet the quality of intake and type of foods may be impaired ${ }^{(24)}$.

The results of clinical evaluation overestimated the instrumental evaluation, similar to the report of Leder ${ }^{(10)}$, since the classification of dysphagia based on the DOSS scale was considered grade 6 for more than half of individuals (64.71\%), i.e. with functional limitations. Conversely, several authors observed that the number of patients after the late stage of stroke with dysphagia increases after instrumental evaluation ${ }^{(9,25)}$, identifying those without signs on clinical evaluation. Several factors may contribute to the divergent results found in the present study and in the literature, such as the different age range ${ }^{(6,11)}$, stage of stroke ${ }^{(10-11)}$ and motor and cognitive impairment ${ }^{(13,14)}$, as well as different evaluation procedures, such as the use of videofluoroscopy ${ }^{(9,10,25)}$.

Evaluation of the quality of life related to swallowing using the SWAL-QOL protocol demonstrated that most individuals presented maximum score close or equal to 100 points, and few had scores close to the 
expected minimum, both concerning the scores of each domain and the global score. No studies were found in the literature applying the SWAL-QOL protocol in individuals affected by stroke in the late stage, yet in laryngectomized individuals ${ }^{(13)}$ it was observed that the lowest means and medians were within the domains "duration of feeding", "communication" and "fear to feed", similar to the present study. Conversely, for individuals with Parkinson disease ${ }^{(14)}$, there is a reduction in scoring in the protocol with the disease, evolution, especially in the domains "burden", "duration of feeding", "communication", "social function", "sleep" and "fatigue".

Most individuals in the present study were classified as presenting mild oropharyngeal dysphagia oropharyngeal swallowing with functional limitations; however, it is believed that the quality of life is an important factor to be investigated, regardless of the degree of oropharyngeal dysphagia, since the different standpoints of each individual should be considered ${ }^{(26)}$.

The scores of the SWAL-QOL protocol were correlated with the degree of oropharyngeal dysphagia classified both by clinical and instrumental evaluation, and it was possible to observe statistically significant correlation in both. Thus, the worse the degree of dysphagia, the greater the impact on the quality of life of these individuals.

The present results agree with the literature even when studies were conducted using other quality of life protocols, such as the BD/ protocol (Beck depression inventor) ${ }^{(27)}, S F 36^{(26)}$ and the SS-QOL scale (Stroke Specific Quality of Life Scale)(27), and according to all authors, in the case of oropharyngeal dysphagia, quality of life is an important aspect to be investigated, since it is altered regardless of the degree of dysphagia.

It was o possible to observe correlation between some specific domains of the SWAL-QOL protocol both by clinical and instrumental evaluation, such as the domains: burden, duration of feeding, wish to feed, frequency of symptoms and mental state. All these domains were related with at least one texture, yet more frequently with solid food. Greater damages to feeding with solid texture may be explained by the need to better prepare the food bolus, which demands a good oral stage of swallowing.

Aging may evolve with tooth loss and utilization of dentures, which are not always well adapted, leading to greater masticatory and swallowing difficulties. Thus, the degree of dysphagia and the oral ingestion level are influenced by the oral health condition ${ }^{(28)}$.

Based on the present results, it may be observed that oropharyngeal dysphagia, even mild, influenced the quality of life related to swallowing in elderly individuals after the late stage of stroke in the global score and specific domains such as burden, duration of feeding, wish to feed, frequency of symptoms and mental state. Thus, the greater the swallowing disorder, the greater was the burden, an overall indicator of daily difficulties experienced by patients with swallowing disorder. Also, the greater was the lack of wish to feed, there were more symptoms of oropharyngeal alterations and more disorders in mental stage, 
identified as self-image problems and psychological suffering ${ }^{(29)}$ in elderly individuals affected by the late stage of stroke in this study.

Even though videofluoroscopy is considered the most effective gold standard method to evaluate and diagnose the oropharyngeal dysphagia, in the present study the accomplishment of nasoendoscopy also

allowed application of a validated classification scale ${ }^{(22)}$. Other study limitation to be considered concerns the fact that the Brazilian Portuguese version of the SWAL-QOL protocol was applied to oncological patients in the process of translation and transcultural adaptation ${ }^{(13)}$, while this study evaluated neurological patients, which may have impaired the analysis or even the manner through which the patients responded to the protocol.

The lack of information on the type of stroke and affected areas represent a limitation of the study, since the stroke may affect several areas of the central nervous system besides the brainstem, and the severity of dysphagia is related to the neurological characteristics of patients $(6,7,14,15)$.

The lack of studies correlating the SWAL-QOL protocol with swallowing dysfunction in individuals affected by the late stage of stroke impairs the comparison with the literature. Thus, further studies are necessary to investigate the quality of life related to swallowing in the different stages of stroke.

Also, it is important to longitudinally follow these individuals concerning the feeding aspects.

\section{Conclusion}

There was association between the severity of oropharyngeal dysphagia and the quality of life of elderly individuals in the late stage of stroke, demonstrating the importance of longitudinal follow-up of these individuals concerning the feeding aspects.

\section{List Of Abbreviations}

- SWAL-QOL: Quality of Life in Swallowing Disorders.

- DOSS: Dysphagia Outcome and Severity Scale.

- BDI protocol: Beck depression inventor.

- SF-36: Medical Outcomes Study 36-Item Short - Form Health Survey

- SS-QOL scale: Stroke Specific Quality of Life Scale

\section{Declarations}

\section{Ethics approval and consent to participate}

This retrospective cross-sectional study was conducted by analysis of a databank of a Speech-Language Pathology Clinic, and was initiated after approval by the Institutional Review Board of Bauru School of 
Dentistry, University of São Paulo, protocol n. 553.710 and CAEE n. 16365913.0000.5417.

\section{Consent por publication}

Not applicable.

\section{Availability of data anda materials}

The datasets generated and/or analysed during the current study are not publicly available due why the medical records are physically archived in the speech therapy clinicbut are available from the corresponding author on reasonable request.

\section{Competing interests}

The authors declare that we have no competing interests.

\section{Funding}

No funding was received.

\section{Authors' contributions}

No applicable.

\section{Acknowledgements}

No applicable.

\section{References}

1. Clavé P, Shaker R. Dysphagia: current reality and scope of the problem. Nat Rev Gastroenterol Hepatol. 2015;12(5):259-70.

2. Rodrigues $T$, Jacuviske $M$, Zazo K. Perfil populacional de pacientes com distúrbios da comunicação humana decorrentes de lesão cerebral, assistidos em hospital terciário. CEFAC. 2011 Mar-Abr; 13(2):330-339.

3. Barros A, Fabio S, Furkim A. Correlação entre os achados clínicos da deglutição e os achados da tomografia computadorizada de crânio em pacientes com acidente vascular cerebral isquêmico na fase aguda da doença. Arq. Neuropsiquiatr. 2006;64(4):1009-14.

4. Crary M, Carnaby-Mann G, Miller L, Antonios N, Silliman S. Dysphagia and Nutritional Status at the Time of Hospital Admission for Ischemic Stroke. J Stroke Cerebrovasc Dis.2006;15(4):164-171.

5. Hammond C, Goldstein L, Horner R, Ying J, Gray L, Gonzalez L, Bolser C. Predicting Aspiration in Patients With Ischemic Stroke: Comparison of Clinic Signs and Aerodynamic Measures of Voluntary Cough. Chest. 2009(3);135;769-777. 
6. Hasan Z, Al-Shimmery E, Taha M. Evaluation of neurogenic dysphagia in Iraqi patients with acute stroke. Neurosciences 2010;15(2):90-96.

7. Antonios N, Carnaby-Mann G, Crary M, Miller L, Hubbard H, Hood K, Sambandam R, Xavier A, Silliman S. Analysis of a Physician Tool for Evaluating Dysphagia on an Inpatient Stroke Unit: The Modified Mann Assessment of Swallowing Ability. J Stroke Cerebrovasc Dis. 2010;19(1):49-57.

8. Martino R, Foley N, Bhogal S, Diamant N, Speechley M. Dysphagia After Stroke: Incidence, Diagnosis, and Pulmonary Complications. Stroke 2005;36(12):2756-2763.

9. Nishiwaki K, Tsuji T, Liu M, Hase K, Tanaka N, Fujiwara T. Identification of a simple screening tool for dysphagia in patients with stroke using factor analysis of multiple dysphagia variable. J Rehabil Med. 2005;37(4):247-251

10. Leder S, Espinosa J. Apiration risk after acute stroke: Comparison of clinical examination and fiberoptic endoscopic evaluation of swallowing. 2002(17):214-218.

11. Mann G, Hankey G, Cameron D. Swallowing Disorders following acute stroke: prevalence and diagnostic accuracy. Cerebrovasc Dis. 2000;10(5):380-6.

12. Mchorney C, Robbins J, Lomax K, Rosenbek J, Chignell K, Kramer A, Bricker D. The SWAL-QOL and SWAL-CARE outcomes tool for oropharyngeal dysphagia in adults: III. Documentation of reliability and validity. Dysphagia.2002;17(2):97-114.

13. Queija D, Portas J, Dedivitis R, Lehn C, Barros A. Deglutição e qualidade de vida após laringectomia e faringolaringectomia total. Braz. j. otorhinolaryngol.2009;75(4), 556-64.

14. Menezes D. Estudo da qualidade de vida em deglutição de parkinsonianos. Arq. NeuroPsiquiatr.2011;(2b):69.

15. Tabor L, Gaziano J, Robinson R, Plowman E. Defining Swallowing-Related Quality of Life Profiles in Individuals with Amyotrophic Lateral Sclerosis. Dysphagia, 2016, pp 1-7.

16. Moon J, Hahm S, Won Y, Cho H. The effects of tongue pressure strength and accuracy training on tongue pressure strength, swallowing function, and quality of life in subacute stroke patients with dysphagia: a preliminary randomized clinical trial. Int J Rehabil Res. 2018;41(3):204-10.

17. Hong D, Yoo D. A comparison of the swallowing function and quality of life by oral intake level in stroke patients with dysphagia. J Phys Ther Sci. 2017;29(9):1552-4.

18. Meerwaldt J. Spatial disorientation in right-hemisphere infarction: a study of the speed of recovery. J Neurol Neurosurg Psychiatry. 1983;46:426-429.

19. Almeida O. Mini Exame do estado Mental e o diagnóstico de demência no Brasil. Arquivos de NeuroPsiquiatria. 1998;56(3B):605-612.

20. Lourenço R, Veras R. Mini -Exame do Estado Mental: características psicométricas em idosos ambulatoriais. Rev Saúde Pública. 2006;40(4):712-719.

21. Silva R. Disfagia neurogênica em adultos: uma proposta para avaliação clínica. In: Furkin AM, Santini CS. Disfagias Orofaríngeas. $2^{\text {a }}$ Ed. Barueri: Pró Fono; 2004. p.35-48. 
22. O’Neil K, Purdy M, Falk J, Gallo L. The Dysphagia Outcome and Severity Scale. Dysphagia, 1999, v.14, p.139-45.

23. Mchorney C, Bricker D, Kramer A, Rosenbek J, Robbins J, Chignell K, Logemman J, Clarke C. The SWAL-QOL Outcomes Tool for Oropharyngeal Dysphagia in Adults: I.Conceptual Foundation and Item Development. Springer-Verlag New York Inc. 2000, Dysphagia 15:115-121.

24. Medin J, Windahl J, Von Arbin M, Tham K, Wredling R. Eating difficulties among patients 3 months after stroke in relation to the acute phase. J.Adv.Nurs. 2012 Mar;68(3): 580-9.

25. Schelp A, Cola P, Gatto A, Silva V, Carvalho L. Incidência de disfagia orofaríngea após acidente vascular encefálico em hospital publico de referência. Arq.Neuropsiquiatr 2004; 62(2-B):503-50.

26. Brandão D, Nascimento J, Vianna L. Capacidade funcional e qualidade de vida em pacientes idosos com ou sem disfagia após acidente vascular encefálico isquêmico. Rev. Assoc. Med. Bras. 2009;55(6):738-743

27. Kang J, Park R, Lee S, Kim J, Yoon S, Jung K, M.D. The Effect of Bedside Exercise Program on Stroke Patients with Dysphagia. Ann Rehabil Med. 2012 August; 36(4): 512-520.

28. Mituuti C, Bianco V, Bentim C, Andrade E, Rubo J, Berretin-felix G. Influence of oral health condition on swallowing and oral intake level for patients affected by chronic stroke. Clinical Interventions in Aging. 2015;(10): 29-35.

29. Mchorney C.A, Bricker D.E, Kramer A.E, Rosenbek J.C, Robbins J.A, Chignell K.A, Logemman J.A, Clarke C. The SWAL-QOL Outcomes Tool for Oropharyngeal Dysphagia in Adults: I.Conceptual Foundation and Item Development. Springer-Verlag New York Inc. 2000, Dysphagia 15:115-121 (2000).

\section{Tables}


Table 1. Minimum, maximum, median, mean and standard deviation values of the different domains of the SWAL-QOL swallowing quality of life protocol $(\mathrm{N}=30)$

\begin{tabular}{llllll}
\hline Domains & Minimum & Maximum & Median & Mean & Standard deviation \\
\hline Burden & 0 & 100 & 100 & 79,58 & 32,07 \\
\hline Eating desire & 0 & 100 & 100 & 69,72 & 31,60 \\
\hline Eating duration & 0 & 100 & 100 & 60,42 & 43,06 \\
\hline Frequency of symptons & 32,14 & 100 & 87,5 & 76,94 & 18,80 \\
\hline Food selection & 12,5 & 100 & 87,5 & 88,33 & 21,00 \\
\hline Communication & 0 & 100 & 62,5 & 71,67 & 32,48 \\
\hline Fear to eat & 0 & 100 & 56,25 & 72,08 & 30,34 \\
\hline Mental health & 0 & 100 & 100 & 78,5 & 33,11 \\
\hline Social & 30 & 100 & 100 & 89,33 & 22,07 \\
\hline Sleep & 0 & 100 & 50 & 67,08 & 33,39 \\
\hline Fatigue & 0 & 100 & 83,33 & 79,71 & 25,96 \\
\hline Total & 40,34 & 98,29 & 79,54 & 77,09 & 16,24 \\
\hline
\end{tabular}

Table 2. Correlation between swallowing quality of life and the classification of the degree of dysphagia by clinical and instrumental assessment (videoendoscopy) of swallowing $(\mathrm{N}=30)$

\begin{tabular}{|c|c|c|c|c|c|c|c|c|}
\hline \multirow{3}{*}{$\begin{array}{l}\text { Quality of life } \\
\text { Domains }\end{array}$} & \multicolumn{6}{|c|}{ Clinica Evaluation } & \multicolumn{2}{|c|}{ Instrumental Evaluation } \\
\hline & \multicolumn{2}{|c|}{ Liquid } & \multicolumn{2}{|c|}{ Pudding } & \multicolumn{2}{|c|}{ Solid } & \multirow[b]{2}{*}{$\mathbf{r}$} & \multirow[b]{2}{*}{$\mathbf{p}$} \\
\hline & $\mathbf{r}$ & $\mathbf{p}$ & $\mathbf{r}$ & $\mathrm{p}$ & $\mathbf{r}$ & $\mathbf{p}$ & & \\
\hline Burden & $-0,27$ & 0,14 & $-0,33$ & 0,07 & $-0,50$ & $<0,01^{*}$ & 0,42 & $<0,01^{*}$ \\
\hline Eating desire & $-0,31$ & 0,09 & $-0,44$ & $<0,01 *$ & $-0,29$ & 0,10 & 0,46 & $<0,01 *$ \\
\hline Eating duration & $-0,19$ & 0,31 & $-0,30$ & 0,10 & $-0,47$ & $<0,01 *$ & 0,20 & 0,28 \\
\hline Frequency of symptons & $-0,47$ & $<0,01^{*}$ & $-0,54$ & $<0,01 *$ & $-0,58$ & $<0,01 *$ & 0,54 & $<0,01 *$ \\
\hline Food selection & 0,22 & 0,24 & 0,07 & 0,68 & $-0,12$ & 0,50 & 0,08 & 0,66 \\
\hline Communication & 0,15 & 0,40 & $-0,22$ & 0,23 & $-0,06$ & 0,71 & 0,31 & 0,09 \\
\hline Fear to eat & $-0,004$ & 0,98 & 0,01 & 0,93 & $-0,10$ & 0,56 & $-0,14$ & 0,44 \\
\hline Mental health & $-0,24$ & 0,19 & $-0,31$ & 0,08 & $-0,43$ & $<0,01^{*}$ & 0,42 & $<0,01 *$ \\
\hline Social & $-0,17$ & 0,36 & $-0,27$ & 0,13 & $-0,25$ & 0,17 & 0,21 & 0,25 \\
\hline Sleep & 0,23 & 0,21 & 0,14 & 0,46 & 0,11 & 0,53 & $-0,18$ & 0,33 \\
\hline Fatigue & $-0,25$ & 0,16 & $-0,11$ & 0,55 & $-0,28$ & 0,13 & 0,13 & 0,50 \\
\hline Total & $-0,32$ & 0,08 & $-0,39$ & $<0,01 *$ & $-0,49$ & 0,005 & 0,46 & $<0,01^{*}$ \\
\hline
\end{tabular}




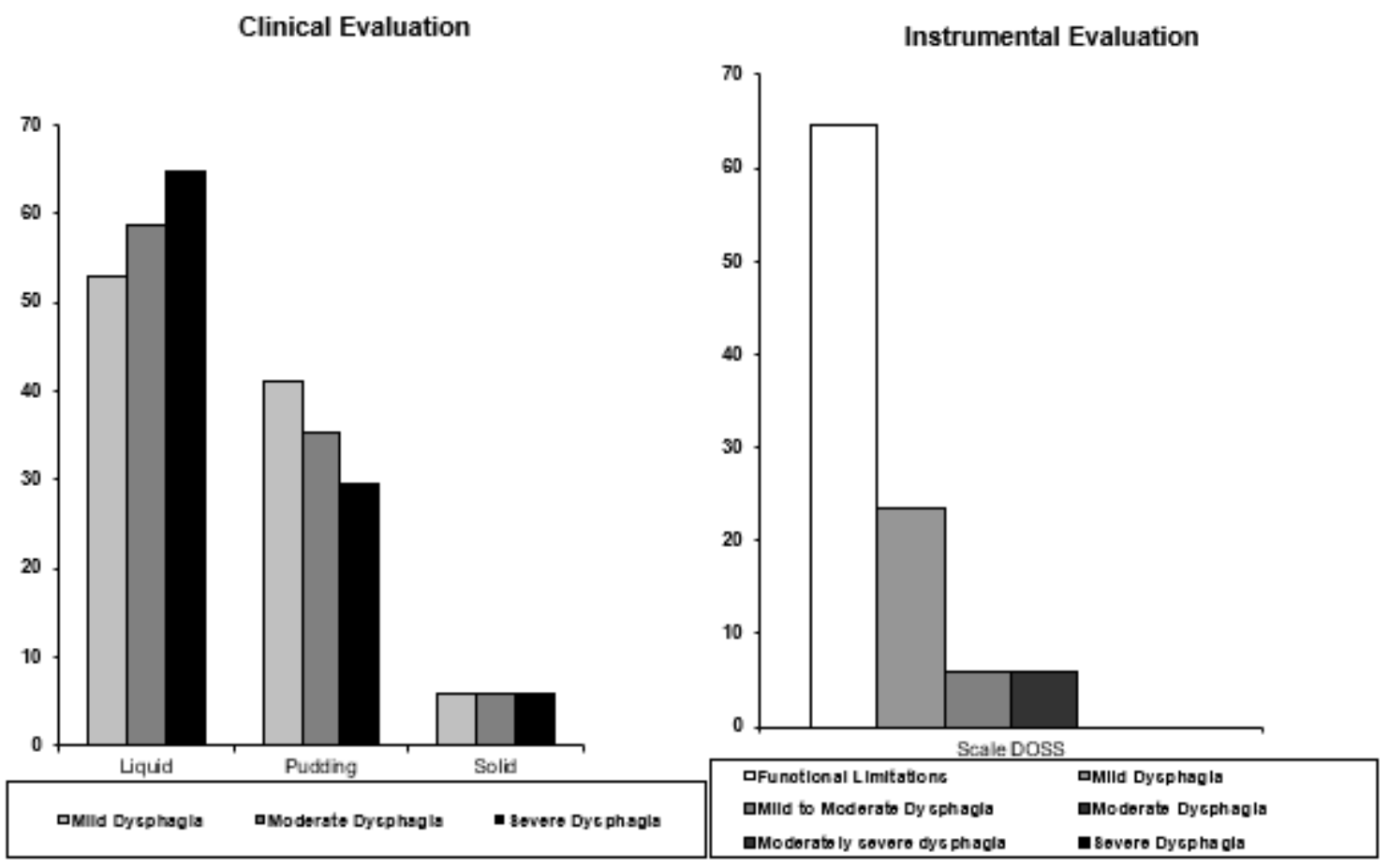

Figure 1

Distribution of the elderly affected by stroke according to the classification of the degree of oropharyngeal dysphagia by clinical and instrumental assessment (videoendoscopy) of swallowing $(\mathrm{N}=$ 30) 